Conclusion: WHODAS 2.0 may not be sensitive enough in detecting quality of life change in hearing-related disability in our Singapore population.

doi:10.1017/S0022215116005612

\section{ID: IP065}

B-Cell Lymphoma of the Temporal bone:

A rare presentation

Presenting Author: Eu Chin Ho

Eu Chin Ho, Shirish Johari

Tan Tock Seng Hospital

Learning Objectives: To highlight the need to be vigilant for unusual diagnoses that may mimic common otological conditions.

Introduction: To report a case of temporal bone B cell lymphoma.

Methods: Review of inpatient case notes of patient with atypical presentation of lymphoma.

Results: Temporal bone involvement by malignancies, primary or secondary, is rare. Diagnosis is frequently delayed as the symptoms mimic more common otological conditions like otitis external or media. Involvement of cranial nerves or cochleovestibular system may occur in advanced cases. More commonly, the bone involvement is due to direct extension of locally invasive squamous or adenocarcinomas of ear. Primary B cell bone lymphomas are also a rare entity and the presentation is usually with pain, swelling or fracture of involved bones. Mastoiditis due to destruction of underlying temporal bone by malignant lymphoma has only been reported in about 20 cases in the literature. We describe a case of primary B cell lymphoma presenting with radiological evidence of mastoiditis, skull base osteomyelitis and sigmoid sinus thrombosis. Patient was initially treated with broad-spectrum antimicrobials and it was not until development of facial palsy that a decision of mastoid exploration was made. Histopathological examination confirmed infiltration of temporal bone by malignant B cells. Staging studies including a diagnostic bone marrow biopsy diagnosed the patient with stage IV primary diffuse large B cell lymphoma of the bone. Treatment was initiated with multi-agent chemotherapy with good results.

Conclusion: Atypical presentation of otological signs and symptoms refractory to medical management requires a thorough evaluation and consideration of uncommon differentials. A high index of suspicion towards temporal bone malignancies may be helpful in timely diagnosis.

doi:10.1017/S0022215116005624

\section{ID: IP066}

Treatment and Outcome of Otitis Media

With Effusion in Cleft Palate Patients
Presenting Author: Yingmin Hoe

Yingmin Hoe

KK Women's and Children's Hospital

Learning Objectives: This study aims to investigate the impact of OME in children with cleft palate.

Objective: Otitis media with effusion (OME) is common in children with cleft palate due to Eustachian tube dysfunction. This study aims to investigate the impact of OME in children with cleft palate.

Study Design: Retrospective case series.

Methods: The case records of patients with cleft palate were analyzed for: type of cleft, age at which repair of cleft palate was performed, age of presentation to the otolaryngologist, age at which Myringotomy and Tympanostomy (M\&T) was done, total number of M\&Ts performed and associated congenital syndromes.

Results: M\&Ts- Out of 600 cleft palate and lip patients, 204 required M\&T. 26.5\% required $\geq 3 \quad \mathrm{M} \& \mathrm{~T}$ and $12.3 \%$ required $\geq 4$ M\&T. $86.8 \%$ of the patients presented early.

Syndromic patients- $15.7 \%$ were syndromic with Pierre Robin being the most common syndrome. They did not require more surgery $(p=0.713)$ nor presented to an Otolaryngologist earlier $(p=0.281)$ than their non-syndromic counterparts.

Type of cleft- $22.1 \%$ had unilateral cleft lip and palate, $32.8 \%$ had bilateral cleft lip and palate, $40.7 \%$ had posterior cleft palate and $4.4 \%$ had submucous cleft palate. Patients with bilateral cleft lip and palate required more M\&Ts than those with posterior and submucous clefts $(p=0.16)$.

Conclusion: OME in cleft palate patients should be actively identified and addressed early, before the development of significant disability. A proportion of patients will have recurrent OME and caregivers should be aware of the need for multiple procedures and long term follow up. Opportunities for combined surgery should be pursued.

doi:10.1017/S0022215116005636

\section{ID: IP067}

Measurement of the Correlation of Implant Stability Quotient with Abutment Length for a Bone Anchored Hearing Implant System

Presenting Author: Morten Høgsbro

Morten Høgsbro

Aalborg University Hospital, Aarhus University Hospital

Learning Objectives: Basic physical principles for the correlation between abutment length and Implant Stability Quotient (ISQ) How these principles translates to a 
laboratory measurement of the correlation of ISQ with abutment length. Implications of the measurements for future studies and clinical measurements.

Introduction: Objective: To quantify the influence from abutment length on measured Implant Stability Quotient (ISQ) for a bone anchored hearing implant system.

Method: Design: Laboratory measurements on temporal bones. The Ponto Wide Implant (Oticon Medical AB, Askim, Sweden) was implanted in temporal bones and measurements of ISQ were made on implant level and for the $6 \mathrm{~mm}, 9 \mathrm{~mm}, 12 \mathrm{~mm}$ and $14 \mathrm{~mm}$ abutments using The Osstell ISQ and SmartPegs (Osstell, Göteborg, Sweden) type 09 (implant level) and type 55 (abutment level). By varying the insertion torque and implantation site, a broad span of implant level ISQ measurements was obtained and compared with the measurements on abutment level. The validity of the data was secured by measuring implant level ISQ before and after measurements on abutment level.

Results: For each abutment length a linear relationship existed between the implant level and the abutment level ISQ throughout the span of ISQs. The slopes for the linear correlations were similar for the different abutments lengths. The relationship for the ISQ as a function of abutment length throughout the span of implant level ISQs was also linear and the slope was measured to be -3.1 ISQ/ $\mathrm{mm} \pm 0.2 \mathrm{ISQ} / \mathrm{mm}$ (standard error of estimate).

Conclusion: The measured correlation between ISQ on implant and abutment level for a bone anchored hearing implant system revealed that the difference in ISQ for different abutment lengths is an additive constant independent of implant level ISQ. This relationship can be used for pooling mean results in clinical studies where different abutment lengths are used.

doi:10.1017/S0022215116005648

\section{ID: IP068}

\section{Evaluation of a New Powerful Sound}

Processor for Bone-Anchored Hearing

Presenting Author: Myrthe Hol

Myrthe Hol, Arjan Bosman, Ivo Kruyt, Emmanuel Mylanus, Ad Snik

Radboudumc

\section{Learning Objectives:}

Introduction: Patients with profound hearing loss for instance as a result of cholesteatoma surgery, may experience problems with air-conduction hearing aids due to tightly fitted ear moulds and/or maximum gain restrictions by acoustic feedback. In profound mixed hearing loss that consists of a moderate sensorineural loss with a large air-bone gap a powerful direct-drive bone-conduction device (BCD) is a viable alternative for a conventional hearing aid, owing to the relatively favourable bone-conduction thresholds.

Until recently, the body-worn Baha Cordelle II processor was the only alternative for patients with a profound mixed hearing loss that needed a BCD. Recently, the head-worn
Cochlear Baha 5 SuperPower Sound Processor (SP5) was introduced, which offers more advanced signal processing and wireless capabilities that may further improve the hearing experience for this patient population. In this study we will compare the performance of both devices.

Objective: We will evaluate the performance of the Baha SP5 relative to the Baha Cordelle II. The objective evaluation comprises aided thresholds, speech perception in quiet and in noise, and loudness growth measures. For the subjective evaluation questionnaires will be used.

Methods: Performance of the Baha SP5 and Baha Cordelle II will be evaluated in a group of 10 experienced Baha Cordelle users. Measures comprise free-field aided thresholds and speech perception in quiet with standard Dutch CVC monosyllables and speech perception in noise with the digits-innoise test. Additionally, loudness growth will be measured for both devices. The performance of either device in real life will be evaluated with APHAB, SSQ, and proprietary questionnaires. The efficacy of wireless sound transmission with Baha SP5 when using the telephone or watching TV will be evaluated with a proprietary questionnaire.

Results: of this study will become available early Spring 2016. Results will be presented at the conference.

doi:10.1017/S002221511600565X

\section{ID: IP069}

Systematic review: the radiological and histological evidence of cochlear trauma following implant insertion

\section{Presenting Author: Emma Hoskison}

Emma Hoskison ${ }^{1}$, Scott Mitchell ${ }^{2}$, Emilie Harterink ${ }^{3}$, Chris Coulson ${ }^{4}$

${ }^{1}$ City Hospital, Birmingham, ${ }^{2}$ Russells Hall

Hospital, Dudley, ${ }^{3}$ Queen Elizabeth Hosptial, Birmingham, ${ }^{4}$ Queen Elizabeth Hospital, Birmingham

Learning Objectives: A systematic review to assess the radiological and histological evidence of cochlear trauma following cochlear implant insertion.

Introduction: Cochlear implantation (CI) has developed from its origins in the $1980 \mathrm{~s}$. Initially, CI was for profound bilateral hearing impairment. However, as candidacy for CI has become more relaxed, there is an increasing emphasis on hearing preservation.

Evidence supports the position that full electrode insertion in an atraumatic fashion into the scala tympani (ST) provides optimal hearing outcomes (Ashendorff et al 2005, Shepherd 1993, Finley et al 2008).

The main aim of this systematic review was to elucidate the degree of trauma associated with CI.

Methods: A systematic literature search was undertaken using PubMed Medline. A grading system described by Eshraghi (2003) was used to classify cochlear trauma. Both radiological and histological studies were included. 